\title{
CHAIN HOMOTOPY AND THE de RHAM THEORY
}

\author{
V. K. A. M. GUGENHEIM AND D. C. SPENCER ${ }^{1}$
}

Introduction. This note contains a method for constructing chainhomotopy operators suitable for the de Rham cohomology theory. In particular, it is proved that differentiably homotopic maps induce chain homotopic chain-mappings in the exterior algebra of differential forms (Formula 13 below; cf. pp. 80-81 of [1], where the same formula is obtained). This shows that the de Rham theory satisfies the "homotopy axiom" in the sense of S. Eilenberg and N. E. Steenrod (cf. [2]); hence the de Rham cohomology groups of a differentiably contractible manifold are trivial. This fundamental result is of ten referred to as the "Poincaré Lemma."

A simple generalization is given in the case of an almost product structure.

Almost complex and complex structures are investigated in $\S 5$; no genuine chain-homotopies are obtained, and in $\$ 6$ is given an example which shows that $\bar{\partial}$-cohomology does not satisfy the homotopy axiom, even in the case of complex manifolds and analytic homotopies; this example is due to Professor K. Kodaira.

1. Definitions and notations. By "manifold" we mean "differentiable manifold of class $C^{\infty}$," by "map," "map of class $C^{\infty}$," etc.; and all notions such as tangent vector or differential form will be taken in their $C^{\infty}$-sense. Tangent vectors will always be taken to have been defined by the $C^{\infty}$-analogue of the definition given in $\S I V$, Chap. II of $[10]$.

If $U$ is a manifold, we denote by $T^{1}(U)$ the tangent bundle, by $T(U)=\sum_{p=0}^{\infty} T^{p}(U)$ the bundle of exterior algebras of tangent vectors. Note that $T^{0}(U)=R=$ the reals. By $\Phi(U)=\sum_{p=0}^{\infty} \Phi^{p}(U)$ we denote the exterior algebra of differential forms; for our purposes, the most convenient definition is

$$
\Phi^{p}(U)=\operatorname{Hom}_{R(U)}\left[\times T^{p}(U), R(U)\right]
$$

where $R(U)=\Phi^{0}(U)=R$-module of $C^{\infty}$-maps $U \rightarrow R$, and $\times T^{p}(U)$ denotes the $R(U)$-module of cross-sections of $T^{p}(U)$. \{ If $\Lambda$ is a commutative ring and $A, B$ are $\Lambda$-modules, $\operatorname{Hom}_{\Lambda}(A, B)$ denotes the $\Lambda$-module of $\Lambda$-homomorphisms $A \rightarrow B$. $\}$

If $v, v^{\prime} \in \times T^{p}(U)$ are such that $v\left|V=v^{\prime}\right| V\left(v=v^{\prime}\right.$ "on $\left.V^{\prime \prime}\right)$ where $V$

Received by the editors December 15, 1954 and, in revised form, March 24, 1955.

1 This work was done while Dr. Gugenheim was a Commonwealth Fund Fellow. 
is some open set of $U$, it is easy to see that $\phi v=\phi v^{\prime}$ on $V$ for $\phi \in \Phi^{p}(U)$. Hence the definition of $\Phi^{p}(U)$ is a "local" one; and $\phi \in \Phi^{p}(U)$ can be given by giving its values on germs of cross-section; a germ of crosssection at $x \in U$ is the equivalence class of all cross-sections which agree (pairwise) in some neighborhood of $x$.

If $\phi \in \Phi^{p+q}(U)$ and $v \in X T^{p}(U)$ we define the contraction $\left.v.\right\rfloor \phi$ $\in \Phi^{q}(U)$ by

$$
(v\lrcorner \phi) v^{\prime}=\phi\left(v \wedge v^{\prime}\right)
$$

where $v^{\prime} \in \times T^{q}(U)$.

The exterior derivative $d: \Phi^{p} \rightarrow \Phi^{p+1}$ is given by the formula

$$
\begin{aligned}
(d \phi)\left(v_{1} \wedge\right. & \left.\cdots \wedge v_{p+1}\right)=\sum_{i=1}^{p+1}(-1)^{i+1} v_{i}\left(\phi\left(v_{1} \wedge \cdots \hat{\imath} \cdots \wedge v_{p+1}\right)\right) \\
& +\sum_{i<j^{7}}(-1)^{i+i+1} \phi\left(\left[v_{i}, v_{j}\right] \wedge v_{1} \wedge \cdots \hat{\imath} \cdots \hat{j} \cdots \wedge v_{p+1}\right)
\end{aligned}
$$

where the $v_{i}$ are germs of $\times T^{1}(U), \phi \in \Phi^{p}(U),\left[v_{i}, v_{j}\right]=v_{i} v_{j}-v_{j} v_{i}$ and $\ldots \hat{\imath} \ldots$ denotes the omission of the term with index $i$. The following will be useful:

LEMMA 1. The homomorphism $d$ is uniquely characterized by:

(i) If $\phi \in \Phi^{0}(U), v \in \times T^{1}(U),(d \phi) v=v \phi$,

(ii) If $\phi \in \Phi^{0}(U), d^{2} \phi=0$,

(iii) If $\phi \in \Phi^{p}(U), \psi \in \Phi(U), d(\phi \wedge \psi)=d \phi \wedge \psi+(-1)^{p} \phi \wedge d \psi$.

Since locally ${ }^{2} \Phi^{1}(U)$ is (isomorphic to) the Grassmann algebra generated by $\Phi^{1}(U)$ regarded as an $R(U)$-module, (ii) and (iii) imply (ii'): $d^{2}=0$.

If $U, V$ are manifolds, and $f: U \rightarrow V$ is a map, we denote by $f_{*}: T(U)$ $\rightarrow T(V)$ and $f^{*}: \Phi(V) \rightarrow \Phi(U)$ the corresponding induced maps.

If $c$ is a differentiable (i.e., $\left.C^{\infty}\right) p$-chain in $U$ and $\phi \in \Phi^{p}(U)$, we shall write $\phi \cdot c=\int_{c} \phi$. Stokes's theorem then takes the form $(d \phi) \cdot c$ $=\phi \cdot b c$, where $b$ denotes the boundary operator of the singular theory.

2. Almost product structure. We say that the manifold $U$ has almost product structure $(P, Q)$ if there are homomorphisms ${ }^{3} P, Q$ : $T^{1}(U) \rightarrow T^{1}(U)$ such that $T^{1}(U)=P T^{1}(U) \oplus Q T^{1}(U)$ (direct sum). Thus for $v \in T^{1}(U), v=P v+Q v$ and hence if $v_{i} \in T^{1}(U)(i=1, \cdots, p)$, then $v_{1} \wedge \cdots \wedge v_{p}$ is a sum of terms each of which is the exterior

2 More accurately: $\Phi(V)$ is the Grassmann algebra generated by $\Phi^{1}(V)$ for sufficiently small neighbourhoods $V \subset U$.

- For fibre bundles the fibres of which are modules, a homomorphism is a fibrepreserving map which, restricted to any fibre, is a homomorphism in the algebraic sense. 
product of $r$ vectors of type $P v_{i}$ by $s$ vectors of type $Q v_{j}$ where $r+s=p$; for given $r, s$ such a term is called a vector of "type $(r, s)$ "; and this process defines a unique projection operator

$$
\prod_{r, s}: T^{r+s}(U) \rightarrow T^{r+s}(U)
$$

onto the submodule of vectors of type $(r, s)$.

We then define projection operators $\prod_{r, s}^{*}: \Phi^{r+s}(U) \rightarrow \Phi^{r+s}(U)$ by $\prod_{r, s}^{*} \phi=\phi \prod_{r, s}$. If $\phi=\prod_{r, s}^{*} \phi$ we say that $\phi$ is of type $(r, s)$ (cf. [8]).

Let $U, V$ be manifolds with almost product structures $(P, Q)$, $(\bar{P}, \bar{Q})$ respectively. A map $F: U \rightarrow V$ is said to be of type $(l, m)$ (in relation to these structures) if

$$
\bar{\prod}_{r+l, 8+m} F_{*}=F_{*} \prod_{r, s}
$$

A map of type $(0,0)$ is said to be admissible; the same definitions apply to any homomorphisms $T(U) \rightarrow T(V)$ or $\Phi(V) \rightarrow \Phi(U)$.

An examination of formula (2) shows that $d=d_{2}^{\prime}+d_{1}^{\prime}+d_{1}^{\prime \prime}+d_{2}^{\prime \prime}$ where $d_{2}^{\prime}, d_{1}^{\prime}, d_{1}^{\prime \prime}, d_{2}^{\prime \prime}$ are of types $(2,-1),(1,0),(0,1),(-1,2)$ respectively. $d^{2}=0$ leads to the following identities:

$$
\begin{aligned}
d_{2}^{\prime 2} & =d_{2}^{\prime} d_{1}^{\prime}+d_{1}^{\prime} d_{2}^{\prime}=d_{2}^{\prime} d_{1}^{\prime \prime}+\left(d_{1}^{\prime}\right)^{2}+d_{1}^{\prime \prime} d_{2}^{\prime} \\
& =d_{2}^{\prime} d_{2}^{\prime \prime}+d_{1}^{\prime} d_{1}^{\prime \prime}+d_{1}^{\prime \prime} d_{1}^{\prime}+d_{2}^{\prime \prime} d_{2}^{\prime} \\
& =d_{2}^{\prime \prime} d_{1}^{\prime \prime}+d_{1}^{\prime \prime} d_{2}^{\prime \prime}=d_{2}^{\prime \prime} d_{1}^{\prime}+\left(d_{1}^{\prime \prime}\right)^{2}+d_{1}^{\prime} d_{2}^{\prime}=\left(d_{2}^{\prime \prime}\right)^{2}=0 .
\end{aligned}
$$

In analogy to Lemma 1 , we now define the $R(U)$-homomorphism $d_{P}: \Phi^{p}(U) \rightarrow \Phi^{p+1}(U)$ by

(i) If $\phi \in \Phi^{0}(U)$ and $v \in \times T^{1}(U)$, then

$$
\left(d_{P} \phi\right) v=(P v) \phi .
$$

(ii) If $\phi \in \Phi^{0}(U),\left(d_{P} d+d d_{P}\right) \phi=0$.

(iii) If $\phi \in \Phi^{p}(U)$ and $\psi \in \Phi(U)$,

$$
d_{P}(\phi \wedge \psi)=d_{P \phi} \wedge \psi+(-1)^{p} \phi \wedge d_{P} \psi
$$

It easily follows that

(ii') $d_{P} d+d d_{P}=0$.

It is easily verified that $2 d_{2}^{\prime}+d_{1}^{\prime}-d_{2}^{\prime \prime}$ satisfies these conditions; whence

$$
d_{P}=2 d_{2}^{\prime}+d_{1}^{\prime}-d_{2}^{\prime \prime} .
$$

Writing also

$$
d_{Q}=2 d_{2}^{\prime \prime}+d_{1}^{\prime \prime}-d_{2}^{\prime},
$$


we see that $d=d_{P}+d_{Q}$ and, by symmetry, that $d_{Q}$ is related to $Q$ as $d_{P}$ is to $P$.

Using (3), (4), we see that $d_{P}^{2}=d_{1}^{\prime 2}+2\left(d_{1}^{\prime} d_{1}^{\prime \prime}+d_{1}^{\prime \prime} d_{1}^{\prime}\right)+d_{1}^{\prime \prime 2}$. Hence, noting $d \mid \Phi^{0}(U)=d_{1}^{\prime}+d_{1}^{\prime \prime}$ and appealing to Lemma 1 , we have

LeMMA 2. $d_{P}^{2}=0$ if and only if $d=d_{1}^{\prime}+d_{1}^{\prime \prime}$; i.e., $d_{2}^{\prime}=d_{2}^{\prime \prime}=0$; i.e., $d_{P}=d_{1}^{\prime}, d_{Q}=d_{1}^{\prime \prime}$.

It is not hard to prove that the conditions of Lemma 2 are equivalent to the "integrability" of the given almost product structure in which case we have a local product structure.

3. The / operation. Let $U, V$ be manifolds. An obvious almost product structure is defined on $U \times V$ by regarding $P, Q$ as the (natural) projection operators associated with the direct sum decomposition $T^{1}(U \times V)=T^{1}(U) \oplus T^{1}(V)$. We shall thus regard vector fields in $U, V$ as lying, in an evident manner, in $U \times V$. It is clear that the conditions of Lemma 2 pertain; we write $d_{U}=d_{P}, d_{V}=d_{Q} . d_{U}$ corresponds to "differentiation in $U$ only."

Now, let $\phi \in \Phi^{r+s}(U \times V)$ and let $c$ be a singular $r$-chain in $U$. Then (using a notation due to N. E. Steenrod, cf. [3]) we define $\phi / c \in \Phi^{s}(V)$ by

$$
\left.(\phi / c)(v)(y)=(-1)^{r s}\left[j_{v}^{*}(v\rfloor \phi\right)\right] \cdot c
$$

where $v \in \times T^{*}(V), y \in V$ and $j_{y}: U \rightarrow U \times V$ is the map $x \rightarrow(x, y)$. Then, as is easily seen,

$$
\begin{aligned}
(-1)^{r s} d(\phi / c) & =(-1)^{r(s+1)} d_{V} \phi / c \\
& =(-1)^{r(s+1)}\left[d \phi / c-d_{U} \phi / c\right] .
\end{aligned}
$$

Also, if $v^{\prime} \in \times T^{s+1}(V)$ we have

$$
\begin{aligned}
(-1)^{r(s+1)}\left(d_{U} \phi / c\right)\left(v^{\prime}\right)(y) & \left.=j_{y}^{*}\left(v^{\prime}\right\rfloor d_{U} \phi\right) \cdot c \\
\left.=(-1)^{s+1}\left[d j_{y}^{*}\left(v^{\prime}\right\rfloor \phi\right)\right] \cdot c & \left.=(-1)^{s+1} j_{y}^{*}\left(v^{\prime}\right\rfloor \phi\right) \cdot b c \\
& =(-1)^{r(s+1)}(\phi / b c)\left(v^{\prime}\right)(y) .
\end{aligned}
$$

Hence

$$
d \phi / c-\phi / b c=(-1)^{r} d(\phi / c)
$$

(cf. 2.9 in [3]).

Now assume that $V$ has almost product structure $(P, Q)$ and that $U=U_{P} \times U_{Q}$. Define an almost product structure $(\bar{P}, \bar{Q})$ on $U \times V$ $=U_{P} \times U_{Q} \times V$ by

$$
\begin{aligned}
& \bar{P} T^{1}(U \times V)=T^{1}\left(U_{P}\right) \oplus P T^{1}(V), \\
& \bar{Q} T^{1}(U \times V)=T^{1}\left(U_{Q}\right) \oplus Q T^{1}(V) .
\end{aligned}
$$


In this situation, formula (8) splits up into various components. We discuss one special case, namely that when $c=c^{\prime} \times x_{Q}$ where $c^{\prime}$ is an $r$-chain in $U_{P}$ and $x_{Q}$ is a point of $U_{Q}$ regarded as a 0 -chain. In this case the homomorphism $\phi \rightarrow \phi / c$ is of type $(-r, 0)$ in relation to the almost product structures $(\bar{P}, \bar{Q}),(P, Q)$. By examining $(8)$ in terms of its components we obtain:

$$
\begin{aligned}
d_{2}^{\prime} \phi / c & =(-1)^{r} d_{2}^{\prime}(\phi / c), \\
d_{1}^{\prime \prime} \phi / c & =(-1)^{r} d_{1}^{\prime \prime}(\phi / c), \\
d_{2}^{\prime \prime} \phi / c & =(-1)^{r} d_{2}^{\prime \prime}(\phi / c), \\
d_{1}^{\prime} \phi / c-\phi / b c & =(-1)^{r} d_{1}^{\prime}(\phi / c)
\end{aligned}
$$

from which, using (4) and (5), we obtain

$$
\begin{aligned}
d_{\bar{P}} \phi / c-\phi / b c & =(-1)^{r} d_{P}(\phi / c), \\
d_{\bar{Q}} \phi / c & =(-1)^{r} d_{Q}(\phi / c) .
\end{aligned}
$$

4. Chain homotopies. Let us retain the notations of $\S 3$, let $W$ be a third manifold and $F: U \times V \rightarrow W$ a map. We define $\lambda: \Phi(W) \rightarrow \Phi(V)$ by

$$
\lambda \psi=(-1)^{r+1}\left(F^{*} \psi\right) / c
$$

for $\psi \in \Phi(W)$. Then, using (8) we get

$$
\left(d \lambda+(-1)^{r+1} \lambda d\right) \psi=\left(F^{*} \psi\right) / b c
$$

Now, consider the case when $c: I \rightarrow U$ is a singular 1 -simplex and define $f_{t}: V \rightarrow W$ by $f_{t}(y)=F(c(t), y)$; then $F$ represents a homotopy, and (12) becomes

$$
d \lambda+\lambda d=f_{1}^{*}-f_{0}^{*}
$$

showing that differentiably homotopic maps induce chain-homotopic homomorphisms.

Next, consider the almost product structures introduced in the second part of $\S 3$, and assume that $F$ is admissible (in relation to these structures). The homomorphism $\lambda$ defined by (11) in terms of an $r$-chain $c$ "in $U_{P}$ " will be denoted by $\lambda_{P}$. Using (10), we get

$$
\begin{gathered}
\left(d_{P} \lambda_{P}+(-1)^{r+1} \lambda_{P} d_{P}\right) \psi=F^{*} \psi / b c, \\
d_{Q} \lambda_{P}+(-1)^{r+1} \lambda_{P} d_{Q}=0
\end{gathered}
$$

and finally, in analogy to (13),

$$
d_{P} \lambda_{P}+\lambda_{P} d_{P}=f_{1}^{*}-f_{0}^{*}
$$

in other words: A homotopy consistent with a given almost product 
structure induces chain-homotopies for the operator $d_{P}$; and similarly for $d_{Q}$.

5. Almost complex structure (cf. $[5 ; 6])$. Let $M$ be an $m$-manifold, and let $C T(M)=T(M) \otimes_{R} C$ where $C$ are the complex numbers; and let $C(M)=C$-module of $C^{\infty}$-maps $M \rightarrow C$. We define

$$
C \Phi^{p}(M)=\operatorname{Hom}_{C(M)}\left[\times C T^{p}(M), C(M)\right]
$$

and $C \Phi(M)=\sum_{p=0}^{\infty} C \Phi^{p}(M)$; cf. (1). We also define $d: C \Phi^{p}(M)$ $\rightarrow C \Phi^{p+1}(M)$ by the formal analogue of $(2)$; the definitions of $f_{*}, f^{*}$ are similarly extended. It is clear that the whole "complex" theory is analogous to the "real" theory; also, $C(M)$ is naturally isomorphic to $R(M) \otimes_{R} C, C \Phi^{p}(M)$ to $\Phi^{p}(M) \otimes_{R} C$ and, under this isomorphism, $d$ corresponds to $d \otimes 1$.

We say that $M$ has a complex almost product structure if there are $C(M)$-homomorphisms $P, Q: C T^{1}(M) \rightarrow C T^{1}(M)$ such that $C T^{1}(M)$ $=P C T^{1}(M) \oplus Q C T^{\mathrm{j}}(M), P, Q$ being projections. It is clear that the theory of almost product structures ( $\$ 2$ above) has an exact analogue in this situation: and we take over, without change, the definitions of $\prod_{r, 8}, \prod_{r, s}^{*}$, "type $(r, s), " d=d_{P}+d_{Q}$, complete with Lemma 2.

We say that $M$ has almost complex structure if it has complex almost product structure together with an isomorphism $k: C T^{1}(M)$ $\rightarrow C T^{1}(M)$ such that $k P C T^{1}(M)=Q C T^{1}(M), k Q C T^{1}(M)=P C T^{1}(M)$, $k^{2}=1$. Then $k$ can be extended to $k: C T(M) \rightarrow C T(M)$ (and with a slight abuse of notation!) $k: C \Phi(M) \rightarrow C \Phi(M)$. We write $k v=\bar{v}$, $k \phi=\Phi$. In this case, in accordance with the usual notation, we write $\partial, \bar{\partial}$ for $d_{P}, d_{Q}$. If $\bar{\partial}^{2}=0$ (cf. Lemma 2) the given almost complex structure is called integrable (cf. [5]).

It is well known that if $M$ has almost complex structure and $n$ complex dimensions, then it can be assigned a Hermitian metric (cf. [4, p. 209]) and in terms of this a duality operator $*: C \Phi^{p}(M)$ $\rightarrow C \Phi^{2 n-p}(M)$ and a scalar product $(\phi, \psi)$ for $\psi, \phi \in C \Phi^{p}(M) ; \operatorname{cf}[1 ; 5$; 7; 8]. These operations satisfy

$$
\begin{aligned}
\left(\prod_{r, s}^{*} \phi, \psi\right) & =\left(\phi, \prod_{r, s}^{*} \psi\right) \\
* \prod_{r, s}^{*} & =\prod_{n-r, n-s}^{*} *
\end{aligned}
$$

and also, writing $\vartheta=-* \partial *$,

$$
(\phi, \vartheta \psi)=(\bar{\partial} \phi, \psi)
$$

if $\phi, \psi$ are forms with compact carriers (cf. [5]). We define 


$$
\Delta=2(\vartheta \bar{\partial}+\bar{\partial} \vartheta) .
$$

Now, let $U$ be a subdomain (i.e., an open set) of $M$ such that the closure of $U$ in $M$ is compact. By $\mathcal{L}$ denote the Hilbert space (in terms of the scalar product just introduced) of norm-finite differential forms on $U$ and by $\mathcal{F}$ the space of forms $\phi \in C \Phi(M)$ such that $\bar{\partial} \phi=\vartheta \phi$ $=0$ and $\phi=0$ outside $U$; then $\mathcal{F}$ can be regarded as a subspace of $\mathcal{L}$; we denote by $F: \mathcal{L} \rightarrow \mathcal{F}$ the associated projection operator. There exists a "Green's operator" $G: \mathcal{L} \rightarrow \mathcal{L}$ such that

$$
\Delta G \phi=\phi-F \phi
$$

(cf. [5]).

Define $H, J: \mathcal{L} \rightarrow \mathcal{L}$ by

$$
\begin{aligned}
H & =2 \vartheta(\bar{\partial} G-G \bar{\partial})+F, \\
J & =2 \vartheta G .
\end{aligned}
$$

If the structure is complex, $\bar{\partial}^{2}=0, \vartheta^{2}=0$ and hence $\bar{\partial} \Delta=\Delta \bar{\partial}, \vartheta \Delta=\Delta \vartheta$; hence in this case $\Delta H=0$. If $U$ is a closed, compact manifold, $\bar{\partial} G$ $-G \bar{\partial}=0$ and $H=F$.

In the case of complex euclidean $n$-space, it is trivial that there exists a Green's operator $G$ satisfying $\Delta G \phi=\phi$ and, if $\phi$ has a compact support, $\bar{\partial} G \phi=G \bar{\partial} \phi$. Hence, if we assume that $U$ is an arbitrary subdomain of complex euclidean space, then $H \phi=0$ provided that the support of $\phi$ is compact relative to $U$.

As is easily verified,

$$
\bar{\partial} J+\dot{J} \partial=I-H .
$$

Let $V$ be another almost complex manifold; give to $U \times V$ the natural induced almost complex structure; and by $J_{U}, H_{U}, \bar{\partial}_{U}$ denote the operators on $C \Phi(U \times V)$ associated with $U$. Then, if $c$ is some singular $r$-chain in $U$, define $L: C \Phi(U \times V) \rightarrow C \Phi(V)$ by

$$
L \phi=J_{U} \phi / c \text {. }
$$

It is easily seen that

$$
(-1)^{r+1} \partial L+L \bar{\partial}=L \bar{\partial}_{U}
$$

or, using (20),

$$
\left((-1)^{r+1} \partial L+L \bar{\partial}\right) \phi=\left(I-H_{U}-\bar{\partial}_{U} J_{U}\right) \phi / c .
$$

Notice that, since there is no Stokes's formula in the geometrical sense for $\bar{\partial}$, no formula analogous to (8) can be obtained; similarly, no formulas analogous to (10) seem to exist, as singular chains cannot be closely related to almost complex structure. 
In particular, if $r=0, \bar{\partial}_{U} J_{U} / c=0$ and (23) becomes

$$
(-\bar{\partial} L+L \bar{\partial}) \phi=\left(I-H_{U}\right) \phi / c .
$$

Let $W$ be a third almost complex manifold, $F: U \times V \rightarrow W$ a map such that $\partial F^{*}=F^{*} \bar{\partial}$, let $c=u_{1}-u_{0}$ where $u_{0}, u_{1} \in U$, write $f_{i}(v)$ $=F\left(u_{i}, v\right)$, and $\lambda=L F^{*}: C \Phi(W) \rightarrow C \Phi(V)$. Then, (24) gives

$$
(-\bar{\partial} \lambda+\lambda \partial) \phi=\left(f_{1}^{*}-f_{0}^{*}\right) \phi-\left(H_{U} F^{*} \phi\right)_{1}+\left(H_{U} F^{*} \phi\right)_{0}
$$

where $\left(H_{U} F^{*} \phi\right)_{i}=H_{U} F^{*} \phi \mid u_{i} \times V$.

If $U$ is compact and connected, $H_{U}=F_{U}$ where $F_{U}$ is the projection onto the space of forms satisfying $\vartheta_{U} \phi=\bar{\partial}_{U} \phi=0$, and $\mathcal{F}_{U}^{0}$ (subspace of such forms of degree 0$)$ is isomorphic to $C$; then $\left(H_{U} F^{*} \phi\right)_{1}$ $=\left(H_{U} F^{*} \phi\right)_{0}$ and $(25)$ becomes

$$
-\bar{\partial} \lambda+\lambda \partial=f_{1}^{*}-f_{0}^{*}
$$

6. An example (cf. the Introduction). Let $G$ be the multiplicative group consisting of matrices of the form

$$
z=\left[\begin{array}{ccc}
1 & z_{1} & z_{2} \\
0 & 1 & z_{3} \\
0 & 0 & 1
\end{array}\right]
$$

where $z_{i} \in C$; let $D$ be the (discrete) subgroup consisting of all $z$ such that $z_{i}$ are Gaussian integers. Then $V=G / D$ (i.e., the space of right cosets $z \cdot D$ ) is a homogeneous compact complex manifold (which was first considered by Iwasawa). It is easily seen that (in classical notation) the holomorphic 1 -forms

$$
w_{1}=d z_{1}, \quad w_{2}=d z_{2}-z_{3} d z_{1}, \quad w_{3}=d z_{3}
$$

are right invariant on $G$; they can hence be regarded as holomorphic 1 -forms on $V$. Further, it is not hard to verify that $w_{1}, w_{2}, w_{3}$ generate the $\bar{\partial}$-homology group $H_{\bar{\partial}}^{1,0}(V)$ of forms of type $(1,0)$. Hence

$$
\operatorname{dim} H_{\bar{\partial}}^{\frac{1}{0} 0}(V)=3 .
$$

By the duality theorem of Kodaira-Serre (cf. $[9 ; 11]$ )

$$
\operatorname{dim} H_{\bar{\partial}}^{2,3}(V)=3 .
$$

It is easy to verify that

$$
\begin{aligned}
& \psi_{1}=w_{2} \wedge w_{3} \wedge \bar{w}_{1} \wedge \bar{w}_{2} \wedge \bar{w}_{3}, \\
& \psi_{2}=w_{3} \wedge w_{1} \wedge \bar{w}_{1} \wedge \bar{w}_{2} \wedge \bar{w}_{3}, \\
& \psi_{3}=w_{1} \wedge w_{2} \wedge \bar{w}_{1} \wedge \bar{w}_{2} \wedge \bar{w}_{3}
\end{aligned}
$$


represent linearly independent elements of $H_{\bar{\partial}}^{2,3}(V)$ and hence generate this group.

Now, every $t \in G$ induces the analytic homeomorphism $T_{t}: z \rightarrow t \cdot z$ of $V$ onto itself; obviously each $T_{t}$ is homotopic to the identity. We have

$$
\begin{aligned}
& \left(T_{t}\right) * w_{1}=w_{1}, \\
& \left(T_{t}\right)^{*} w_{2}=w_{2}-t_{3} w_{1}+t_{1} w_{3}, \\
& \left(T_{t}\right) * w_{3}=w_{3}
\end{aligned}
$$

and hence

$$
\begin{aligned}
& \left(T_{t}\right)^{*} \psi_{1}=\psi_{1}+t_{3} \psi_{2} \\
& \left(T_{t}\right)^{*} \psi_{2}=\psi_{2} \\
& \left(T_{t}\right)^{*} \psi_{3}=\psi_{3}-t_{1} \psi_{2}
\end{aligned}
$$

showing that there is no chain-homotopy.

\section{REFERENCES}

1. G. de Rham and K. Kodaira, Harmonic integrals, Princeton, Institute for Advanced Study, 1950 (Polycopied).

2. S. Eilenberg and N. E. Steenrod, Foundations of algebraic topology, Princeton, 1952.

3. N. E. Steenrod, Homology of symmetric groups and reduced power operations, Proc. Nat. Acad. Sci. U.S.A. vol. 39 (1953) pp. 213-217.

4. - The topology of fibre bundles, Princeton, 1951.

5. D. C. Spencer, Potential theory and almost-complex manifolds, Conference on Complex Variables, University of Michigan, June, 1953.

6. C. Ehresmann, Sur les variêtes presque complexes, Proceedings of the International Congress of Mathematicians, Cambridge, Mass. 1950, Providence, American Mathematical Society, 1952, vol. 2, pp. 412-419.

7. G. de Rham, Variêtés différentiables, Paris, Hermann, 1955.

8. P. R. Garabedian and D. C. Spencer, A complex tensor calculus for Kähler manifolds, Acta Math. vol. 89 (1953) pp. 279-331. 9-26.

9. J. P. Serre, Un theorème de dualité, Comment. Math. Helv. vol. 29 (1955) pp.

10. C. Chevalley, Theory of Lie groups, I, Princeton, 1946.

11. K. Kodaira, On cohomology groups of compact analytic varieties with coefficients in some analytic faisceaux, Proc. Nat. Acad. Sci. U.S.A. vol. 39 (1953) pp. 865-868.

\section{Birkbeck College and}

Princeton University 